\title{
Thermally assisted magnetization reversal in nanostructures
}

\author{
D. Hinzke*, U. Nowak, and K. D. Usadel
}

\author{
Theoretische Tieftemperaturphysik, \\ Gerhard-Mercator-Universität Duisburg, \\ 47048 Duisburg/Germany
}

\begin{abstract}
Magnetization reversal in Co nanostructures is simulated over a wide range of time-scales, from fast switching processes on a ps time-scale to thermally activated reversal on a $\mu$ s time-scale. Langevin dynamics is used as well as time quantified Monte Carlo methods for the simulation of a classical spin system modeling elongated Co nano-particles. We study the behavior of the magnetization during the reversal, the energy barriers which are relevant for the thermally activated long-time behavior and the corresponding characteristic times.
\end{abstract}

Keywords: Magnetization reversal, classical spin models, fast switching, nanostructures, thermal activation

\section{Introduction}

Magnetic materials are now controllable on a nanometer length scale and there is a broad interest in the understanding of the magnetism of such small structures in view of industrial applications. Magnetic particles which are small enough to be

\footnotetext{
${ }^{*}$ Corresponding author: e-mail: denise@thp.uni-duisburg.de
} 
in a single-domain remanent state might have good qualities for magnetic recording and are thought to enhance the storage density. Recently, much effort was hence focused on the understanding of magnetization reversal dynamics at finite temperatures since thermal activation plays a crucial role for the stability of the magnetization in nanostructures. It sets the ultimate limit for the density of data storage, the so-called superparamagnetism (Chantrell and O'Grady, 1994).

Throughout this paper, we study magnetic nanostructures numerically, taking into account finite temperatures with the aid of Langevin dynamics (Lyberatos and Chantrell, 1993) as well as Monte Carlo methods (Nowak, Chantrell and Kennedy, 2000). Starting with the deterministic spin dynamics on short time scales which plays a crucial role in high-speed data storage (Leineweber and Kronmüller, 2000) we go on to the probabilistic long-time behavior where the reversal is thermally assisted.

\section{Model and Simulations}

We consider a classical three dimensional Heisenberg Hamiltonian for localized spins

$$
\begin{aligned}
\mathcal{H}= & -J \sum_{\langle i j\rangle} \mathbf{S}_{i} \cdot \mathbf{S}_{j}-\mu_{s} \mathbf{B} \cdot \sum_{i} \mathbf{S}_{i}-d_{z} \sum_{i}\left(S_{i}^{z}\right)^{2} \\
& -w \sum_{i<j} \frac{3\left(\mathbf{S}_{i} \cdot \mathbf{e}_{i j}\right)\left(\mathbf{e}_{i j} \cdot \mathbf{S}_{j}\right)-\mathbf{S}_{i} \cdot \mathbf{S}_{j}}{r_{i j}^{3}}
\end{aligned}
$$

where the $\mathbf{S}_{i}=\boldsymbol{\mu}_{i} / \mu_{s}$ are three dimensional magnetic moments of unit length. The first sum represents the ferromagnetic exchange of the moments where $J$ is the coupling constant, the second sum is the coupling of the magnetic moments to an external magnetic field $B$, the third sum represents a uniaxial anisotropy favoring the $z$ axis as easy axis of the system and the last sum represents the dipolar interaction. $w=\mu_{0} \mu_{s}^{2} /\left(4 \pi a^{3}\right)$ describes the strength of the dipole-dipole interaction with $\mu_{0}=4 \pi \cdot 10^{-7} \mathrm{Vs} /(\mathrm{Am})$. The $\mathbf{e}_{i j}$ are unit vectors pointing from lattice site $i$ to $j$. 
In the following, the Hamiltonian is interpreted as the discretization of a continuum model on a cubic lattice with the lattice constant $a$. The transformation to the material parameters which are usually used in a continuum model is given by $J=2 a A_{x}$ where $A_{x}$ is the exchange energy, $d_{z}=K a^{3}$ where $K$ is the anisotropy energy density and $\mu_{s}=M_{s} a^{3}$ where $M_{s}$ is the spontaneous magnetization.

Throughout this paper, we consider a finite, ellipsoidal Co particle with length $L=4 \mathrm{~nm}$ and diameter $D=2 \mathrm{~nm}$. The corresponding material parameters are $A_{x}=1.3 \cdot 10^{-11} \mathrm{~J} / \mathrm{m}$, $K=6.8 \cdot 10^{5} \mathrm{~J} / \mathrm{m}^{2}$ and $M_{s}=1.401 \cdot 10^{6} \mathrm{~A} / \mathrm{m}$. Our simulation starts with a configuration where all magnetic moments point into the $z$-direction (the easy axis) with the $z$ component of the external magnetic field antiparallel to the magnetization.

In order to compute the dipole-dipole interaction efficiently, we use Fast Fourier Transformation methods for calculating the long range interaction (Yuan and Bertram, 1992, Berkov, Ramstöck and Hubert, 1993). The implementation of the Fast Fourier Transformation (FFT) in the Monte Carlo algorithm was described in details before (Hinzke and Nowak, 2000).

In the present paper, we study the magnetization reversal in two limiting cases, for large magnetic fields in the low damping limit and for small magnetic fields in the high damping limit, respectively. In the first limit spin precession is important which can be taken care of by studying the corresponding Landau-Lifshitz Gilbert equation while in the second case spin relaxation is important which we can study more effectively by using Monte Carlo simulations with quantified time step (Nowak, Chantrell and Kennedy, 2000).

In the low damping limit, we solve numerically the LandauLifshitz-Gilbert equation of motion with Langevin dynamics using the Heun method (García-Palacios and Lázaro, 1998). This equation has the form

$$
\frac{\left(1+\alpha^{2}\right) \mu_{s}}{\gamma} \frac{\partial \mathbf{S}_{i}}{\partial t}=-\mathbf{S}_{i} \times\left(\mathbf{H}_{i}(t)+\alpha\left(\mathbf{S}_{i} \times \mathbf{H}_{i}(t)\right)\right)
$$

with the internal field $\mathbf{H}_{i}(t)=\boldsymbol{\zeta}_{i}(t)-\frac{\partial \mathcal{H}}{\partial \mathbf{S}_{i}}$, the gyromagnetic ratio $\gamma=1.76 \times 10^{11}(\mathrm{Ts})^{-1}$ and the dimensionless damping 
constant $\alpha$. The noise $\boldsymbol{\zeta}_{i}(t)$ represents thermal fluctuations, with $\left\langle\boldsymbol{\zeta}_{i}(t)\right\rangle=0$ and $\left\langle\zeta_{i}^{\eta}(t) \zeta_{j}^{\theta}\left(t^{\prime}\right)\right\rangle=2 \delta_{i j} \delta_{\eta \theta} \delta\left(t-t^{\prime}\right) \alpha k_{\mathrm{B}} T \mu_{s} / \gamma$ where $i, j$ denote once again lattice sites and $\eta, \theta$ Cartesian components.

For the long time investigations, we use a Monte Carlo method with a time step quantification, where one Monte Carlo step per spin (MCS) corresponds to a well-defined time interval of the Landau-Lifshitz-Gilbert equation in the high damping limit and on time-scales well above the time of precession (for details see (Nowak, Chantrell and Kennedy, 2000)). In the following, we use $\alpha=4$ for our Monte Carlo simulations.

\section{Results}

In sufficiently small particles the magnetic moments rotate coherently while overcoming the energy barrier $\Delta E$. The first descriptions of the coherent rotation in elongated single domain particles was developed by Stoner and Wohlfarth (1949). Depending on the angle between the applied field $B$ and the $z$ (easy) axis of the system the coercive field $B_{c}$ varies which can be illustrated with the aid of the Stoner-Wohlfarth asteroid (Stoner and Wohlfarth, 1949). Under an angle of $45^{\circ}$ the coercive field $B_{c}$ is given by $B_{c}=d_{\mathrm{eff}} V / \mu_{\mathrm{s}} \approx 0.7 \mathrm{~T}$ where $V$ is the volume of the particle and $d_{\text {eff }}$ is an effective anisotropy constant.

First, we are interested in fast switching reversal processes where the applied field is higher than the coercive field. In this case the energy barrier vanishes and the reversal process starts immediately. Figure 1 shows the time dependence of the $z$ component of the magnetization of our Co particle with space discretization $a=0.25 \mathrm{~nm}$ in the low damping limit ( $\alpha=0.1$ ). Here, we simulate the system with Langevin dynamics, since we are interested in the observation of spin precession. In this simulation the field $B$ is set under an angle of $45^{\circ}$ to the $z$ axis. The $z$ component of the magnetization shows the precession of magnetic moments. The response of the system to the external 


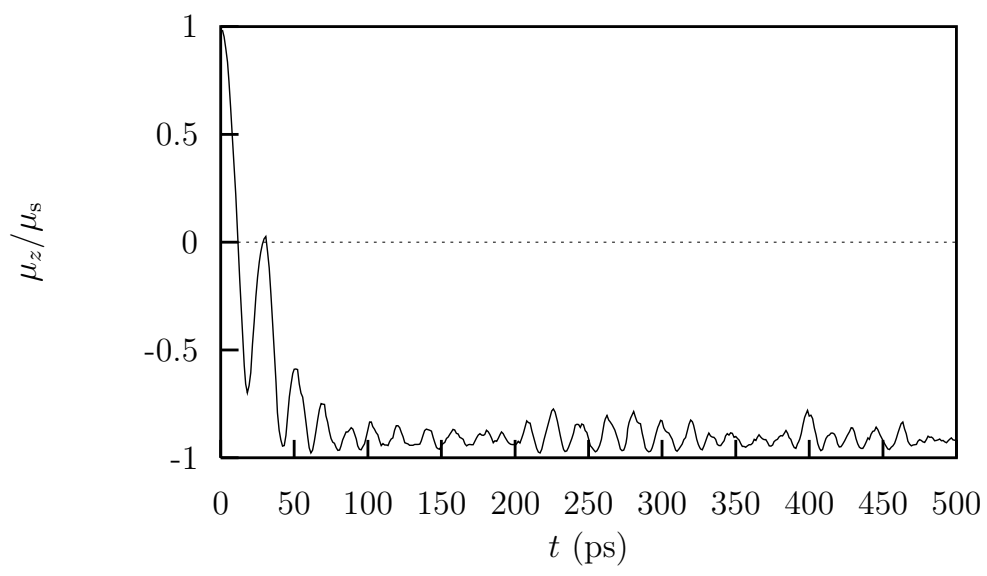

Figure 1: $z$-component of the magnetic moments per cell $\mu_{z}$ vs. time $t$ for a Co system of length $L=4 \mathrm{~nm}$ and diameter $D=2 \mathrm{~nm}$. The data are from Langevin dynamics simulations. $B=1.13 \mathrm{~T}$ and $k_{\mathrm{B}} T=2.21 \cdot 10^{-23} \mathrm{~J}$.

field sets directly in and the magnetization starts oscillating. Note, that the precession time of our system is not simply given by the precession time of a single spin in an external field $\left(\tau_{p}=2 \pi\left(1+\alpha^{2}\right) /(\gamma B) \approx 32 \mathrm{ps}\right.$ in our case). Instead, the whole internal field is relevant for the spin precession, i. e. also contribution from the dipolar field, the exchange and the anisotropy. Note also, that even after the new stable state is reached the magnetization still keeps on oscillating around the equilibrium value, driven by thermal fluctuations.

As yet, we discussed fast switching processes where $B>$ $B_{c}$. Now, we turn to the case $B<B_{c}$, where the reversal process can only occur when it is thermally activated. Figure 2 shows the typical time dependence of the magnetization of our Co particle with space discretization $a=0.5 \mathrm{~nm}$. We are interested in the long time and high damping limit $(\alpha=4)$ so that we can use Monte Carlo simulations. In the following we set the field $B$ antiparallel to the initial state, so that the coercive field is given by $B_{c}=2 d_{\mathrm{eff}} V / \mu_{\mathrm{s}} \approx 1.39 \mathrm{~T}$. First, the magnetization remains nearly constant for some time and 


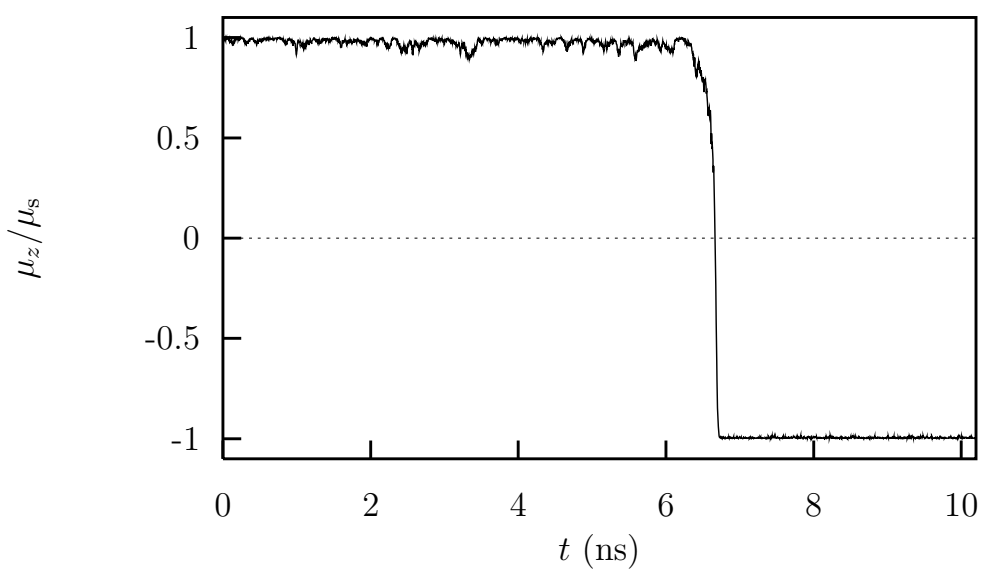

Figure 2: $z$-component of the magnetic moment per cell vs. time. The data are from Monte Carlo simulations. $B=1.1 \mathrm{~T}$ and $k_{\mathrm{B}} T=7.1 \cdot 10^{-23} \mathrm{~J}$.

then, suddenly the value of the $z$ component of the magnetization changes its sign. The value of the corresponding switching time is approximately $6.6 \mathrm{~ns}$. However, this is not a deterministic process as before, where the switching time followed from an equation of motion. Instead, the thermal activation process here is probabilistic and the probability $P\left(t_{s}\right)$ for a reversal after a time $t_{s}$ is given by an exponential law (Brown, 1963)

$$
P\left(t_{s}\right) \sim \exp \left(-t_{\mathrm{s}} / \tau\right),
$$

where $\tau$ is a characteristic time scale. This law is expected to be valid in the range of large time-scales. In such a thermally activated reversal process the corresponding characteristic time is

$$
\tau=\tau_{0} \exp \left(\Delta E /\left(k_{\mathrm{B}} T\right)\right)
$$

where $\tau_{0}$ is a prefactor depending on the system parameters, the temperature, the applied magnetic field and the damping constant. The corresponding energy barrier has the form

$$
\Delta E=d_{\mathrm{eff}} V\left(1-\frac{B}{B_{c}}\right)^{2} .
$$




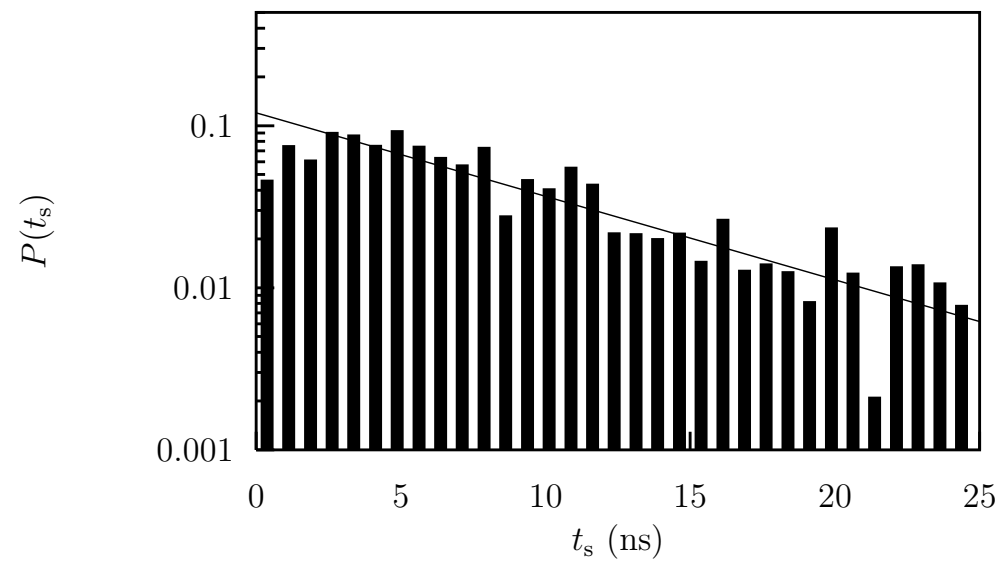

Figure 3: Distribution $P\left(t_{\mathrm{s}}\right)$ vs. switching time $t_{\mathrm{s}}$ for our Co particle at $k_{\mathrm{B}} T=7.1 \cdot 10^{-23} \mathrm{~J}$. The data are from Monte Carlo simulations. The solid line represents the exponential behavior with $\tau=8.43 \mathrm{ps} . B=1.1 \mathrm{~T}$

The prefactor as well as the energy barrier have been calculated by Brown(1963) under the assumption that all magnetic moments are parallel so that the system behaves like one single magnetic moment. In our case, however, the magnetic moments are not strictly parallel so that prefactor and energy barrier are not known offhand.

Figure 3 shows the distribution of switching times from 10000 Monte Carlo runs. The distribution shows an exponential behavior and confirms Eq. 3 with an averaged characteristic time of $8.43 \mathrm{ps}$. As one would expect, on short time-scales, $t_{s} \ll \tau$, the distribution deviates from the simple exponential behavior.

For a further analysis we also extract the energy barrier which is responsible for our reversal process. Figure 4 shows the temperature dependence of the characteristic time following from our simulations. The slope of the straight line corresponds to the energy barrier for a reversal by coherent rotation with its theoretical value $\Delta E=3.27 \cdot 10^{-22} \mathrm{~J}\left(B_{c}=1.39 \mathrm{~T}\right)$ obtained from Eq. 5. Obviously, it is in very good agreement with our numerical data for low enough temperatures. 


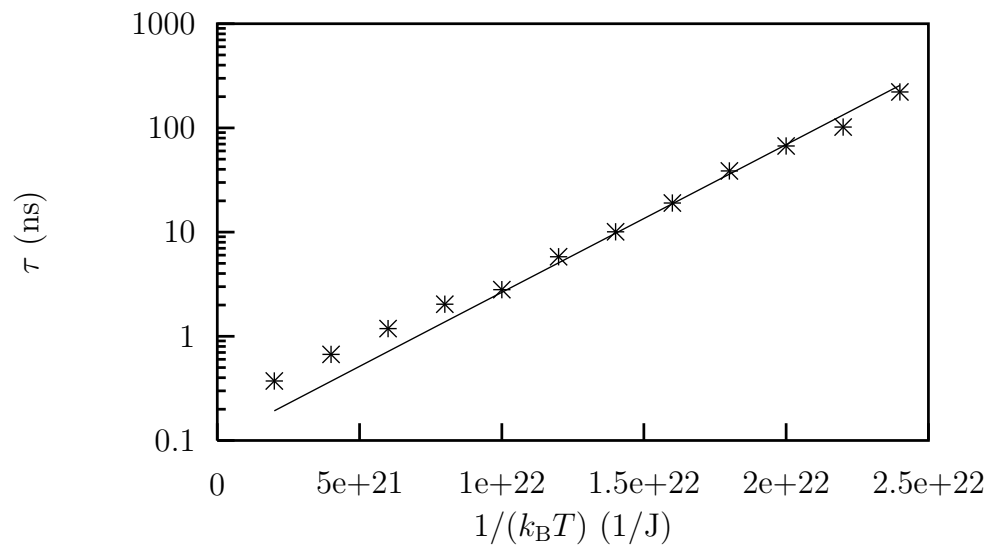

Figure 4: Characteristic time $\tau$ vs. $1 / k_{\mathrm{B}} T$ for a Co system with space discretization $a=0.5 \mathrm{~nm}$. The slope of the solid line represents the energy barrier $\Delta E . B=1.1 \mathrm{~T}$.

\section{Conclusions}

We investigated magnetization reversal processes in singledomain ferromagnetic Co nanoparticles. Depending on the applied field fast switching governed by the Landau-Lifshitz equation of motion with Langevin dynamics can occur or a thermally activated switching processes on a much longer timescale. We studied two limiting cases, fast processes for higher fields and low damping and slow processes for lower fields and high damping. We discussed the influence of spin precession, the distribution of switching times of the thermal activation process and confirm the predicted exponential behavior. We also found good agreement between the energy barrier received from numerical data and theoretical predictions.

\section{Acknowledgments}

This work was supported by the Deutsche Forschungsgemeinschaft. 


\section{References}

Berkov, D. V., Ramstöck, K. R. and Hubert, A. (1993). Solving micromagnetic problems. Phys. stat. sol. (a) 137, 207.

Brown, W. F. (1963). Thermal fluctuations of a single-domain particle. Phys. Rev. 130, 1677.

Chantrell, R. W. and O'Grady, K.: 1994. The magnetic properties of fine particles. in R. Gerber, C. D. Wright and G. Asti (eds), Applied Magnetism. Kluwer Academic Publishers. Dordrecht.

García-Palacios, J. L. and Lázaro, F. J. (1998). Langevindynamics study of the dynamical properties of small magnetic particles. Phys. Rev. B 58, 14937.

Hinzke, D. and Nowak, U. (2000). Monte carlo simulations with dipolar fields calculation by use of fast fourier transformations. J. Magn. Magn. Mat. 221, 365.

Leineweber, T. and Kronmüller, H. (2000). Influence of the intrinsic dynamics of the magnetisation on the hysteresis loop. Physica B 275, 5.

Lyberatos, A. and Chantrell, R. W. (1993). Thermal fluctuations in a pair of magnetostatistically coupled particles. J. Appl. Phys. 73, 6501.

Nowak, U., Chantrell, R. W. and Kennedy, E. C. (2000). Monte Carlo simulation with time step quantification in terms of Langevine dynamics. Phys. Rev. Lett. 84, 163.

Stoner, E. C. and Wohlfarth, E. P. (1949). A mechanism of magnetic hysteresis in heterogeneous alloys. Philos. Trans. R. Soc. London Ser. A 240, 599.

Yuan, S. W. and Bertram, H. N. (1992). Fast adaptive algorithm for micromagnetics. IEEE Trans. Mag. 28, 2031. 